\title{
Combining the Transcorrelated method with Full Configuration Interaction Quantum Monte Carlo: application to the homogeneous electron gas
}

\author{
Hongjun Luo ${ }^{*, \dagger}$ and Ali Alavi*,†, \\ $\dagger$ †ax-Planck-Institut for Solid State Research, Heisenbergstraße 1, 70569 Stuttgart, \\ Germany \\ $\ddagger$ Department of Chemistry, University of Cambridge, Lensfield Road, Cambridge, CB2 \\ $1 E W$, United Kingdom \\ E-mail: h.luo@fkf.mpg.de; a.alavi@fkf.mpg.de
}

\begin{abstract}
We suggest an efficient method to resolve electronic cusps in electronic structure calculations, through the use of an effective transcorrelated Hamiltonian. This effective Hamiltonian takes a simple form for plane wave bases, containing up to two-body operators only, and its use incurs almost no additional computational overhead compared to that of the original Hamiltonian. We apply this method in combination with the full configuration interaction quantum Monte Carlo (FCIQMC) method to the homogeneous electron gas. As a projection technique, the non-Hermitian nature of the transcorrelated Hamiltonian does not cause complications or numerical difficulties for FCIQMC. The rate of convergence of the total energy to the complete basis set limit is improved from $\mathcal{O}\left(M^{-1}\right)$ to $\mathcal{O}\left(M^{-5 / 3}\right)$, where $M$ is the total number of orbital basis functions.
\end{abstract}

PACS numbers: 71.10.Ca, 31.15.V-, 71.15.-m, 02.70.Ss 


\section{Introduction}

Electron correlation can be roughly classified into static correlation and dynamic correlation. In conventional configuration descriptions of the many-body wave function, the two different types of electron correlation are treated in the same way, i.e., by linear expansion in terms of Slater determinants. ${ }^{1]}$ While such a configuration description offers a natural and efficient way to deal with static correlation, it, however, does not treat dynamic correlation efficiently, and thus usually leads to a slow convergence to the complete basis limit (CBL). The main problem is that, due to the Coulomb singularity of the electronic interaction, the shortrange dynamic correlation introduces non-smoothness into the many-body wave function, which can not be approximated efficiently by orbital product expansions. This problem is even more severe for those methods aiming at high accuracy, such as full configuration interaction (FCI) methods and high-order coupled-cluster methods. On the other hand, the non-smoothness of the many-body wave function can be locally resolved and is expressed as the well known Kato cusp condition ${ }^{2}$

$$
\left.\frac{\partial \Psi}{\partial r_{i j}}\right|_{r_{i j}=0}=\left.\frac{1}{2} \Psi\right|_{r_{i j}=0}
$$

Incorporating this property into the construction of many-body wave functions should in principle speed up convergence to the CBL.

One way of incorporating the cusp condition is to introduce explicitly correlated basis functions for the expansion of the many body-wave function. In the last few decades, various

explicit correlation methods (e.g., R12 and F12 methods ${ }^{3}$ ) have been suggested and have achieved a high level of success. The main feature of these methods is that, instead of the conventional approximation of the cusp in terms of orbital product expansions, electron pair geminal functions are directly used in the construction of the basis of the many-body wave function. These geminal functions describe the cusps very efficiently, although they also make the involved calculations highly non-linear. For example, in these calculations 
one has to deal with various kind of orthogonality constraints, which lead to multi-electron integrals (three, four and even higher-body electron integrals). These integrals are usually approximated by resolution of identity (RI) techniques. $\underline{3-5[10}$

Electron cusps can also be efficiently described by using the Jastrow ansatz 11

$$
\Psi(\mathbf{R})=e^{\tau(\mathbf{R})} \Phi(\mathbf{R}), \quad \mathbf{R}=\left(\mathbf{r}_{1}, \mathbf{r}_{2}, \cdots, \mathbf{r}_{N}\right)
$$

where $\Phi$ is an anti-symmetric reference function and $\tau$ is a symmetric pair correlation factor

$$
\tau(\mathbf{R})=\frac{1}{2} \sum_{i \neq j} u\left(\mathbf{r}_{i}, \mathbf{r}_{j}\right)
$$

The correlation factor can be constructed to fulfill the cusp condition (1), and thus the regularity of the reference function $\Phi$ is higher than that of the wave function $\Psi$. Fournais et al. $\stackrel{12}{ }$ have proven that the correlation factor can improve the regularity of the wave function from $C^{0,1}$ to $C^{1,1}$. In Appendix $\mathrm{A}$ we will show that this will lead to a speedup of basis convergence for three dimensional non spin-polarised systems from $M^{-1}$ to $M^{-5 / 3}$. IT In order to guarantee size consistency, the correlation factor has to take an exponential form. This makes the Jastrow ansatz highly non-linear and any variational treatment leads to extremely high-dimensional integrals. Presently the Jastrow ansatz is primarily used in various quantum Monte Carlo methods, such as variational Monte Carlo (VMC) and diffusion Monte Carlo (DMC) methods, $\underline{15-17}$ where the involved integrals can be evaluated directly in the high dimensional space. The exponential correlation factor has also been treated by various expansions, such as the linked cluster expansion, $\frac{18 \mid 19}{19}$ random phase approximations (RPA), $\stackrel{20}{2}$ Fermi hypernetted chain (FHNC) method. .2122 These sophisticated methods are

I It has been found that $C^{1,1}$ is the optimal regularity for the product ansatz, and thus we can only expect a $M^{-5 / 3}$ convergence for the Jastrow ansatz. However, it has been pointed out by Fournais et al. that higher order regularity can be expected for a more general additive ansatz. 13 This is consistent with the early work of Kutzelnigg and Morgen, $\frac{14}{14}$ where, based on such an additive ansatz, different cusp conditions are applied to different types of electron pairs, and thus a higher order convergence ( $\operatorname{such}$ as $M^{-7 / 3}$ ) is achieved. Unfortunately such kind of additive ansatz is not size consistent. 
highly nonlinear and difficult to implement in practical calculations.

For an efficient treatment of the exponential correlation factor, a relatively simple method, the transcorrelated (TC) method, 230 was suggested by Boys and Handy roughly half a century ago. By using a similarity transformation, $e^{-\tau} \hat{H} e^{\tau}$, the exponential correlation factor is removed from the involved equations. The original TC method of Boys and Handy was designed for the single-determinant Jastrow ansatz and contains two equations, for the calculation of the correlation factor $\tau$ and the orbitals respectively. Initial calculations demonstrated that this method can efficiently recover much of the correlation energy. On the other hand the resulting non-Hermitian effective Hamiltonian cannot prevent the energy from falling below the exact one. The lack of a variational bound is considered to a severe problem and has hampered a broad application of the TC method for quite a long time.

Recently, there has been renewed interest in the development of the TC method. Ten-no used the TC Hamiltonian in the perturbation and the coupled electron-pair approximations. ${ }^{[11}$ In this approach, the correlation factor $\tau$ is a fixed local geminal satisfying the cusp condition, while the reference function is treated by conventional configuration expansions. The reference function $\Phi$ is much smoother than the many-body wave function $\Psi$ and, as a consequence, the configuration expansion of $\Phi$ converges much faster. The price to pay is the introduction of a three-body operator in the effective Hamiltonian, as well as various numerical problems due to non-Hermiticity. The non-Hermiticity problem is more severe for self consistent optimization methods. Hino et al. suggested to use biorthogonal basis to deal with the TC Hamiltonian. ${ }^{[32}$ Umezawa et al. optimized the orbitals by minimizing the energy variance. ${ }^{3334}$ Luo has suggested a general variational method for simultaneous optimizations of the correlation factor and the reference function. $\frac{35136}{}$ Yanai et al. have used a truncated canonically transformed Hamiltonian to eliminate the non-Hermiticity of the effective Hamiltonian. $\frac{3738}{}$ Grüneis et al. recently offered a detailed discussion on the choice of correlation factors. ${ }^{39}$

In this work we incorporate the TC method into the full configuration-interaction quan- 
tum Monte Carlo (FCIQMC) method, 4042 aiming at highly accurate calculations on periodic systems. FCI in principle provides the most accurate description of the wavefunction, within an orbital representation, and the results are usually used to benchmark other calculation results. However, FCI methods are also extremely expensive, with the computational cost scaling exponentially with respect to the system size. On the other hand, the extremely large FCI expansion is also very sparse, so that the vast majority of expansion coefficients are essentially zero. This sparsity, however, usually has no regular pattern, especially for strongly correlated systems. The recently developed FCIQMC method and its 'initiator' adaptation $(i-\text { FCIQMC })^{4143}$ offers a way to detect and make use of this sparsity. This method is based on Monte Carlo simulations of the dynamic evolution of the many-body wave function with imaginary time:

$$
\Psi(t)=e^{-t\left(\hat{H}-E_{0}\right)} \Psi(t=0)
$$

which leads to the ground state wave function in the long-time limit $\Psi_{0}=\Psi(t \rightarrow \infty)$. The FCI expansion coefficients are simulated by a set of walkers which evolve over imaginary time. In the long-time limit, a steady distribution of the walkers is reached and the corresponding projection energy, in the large-walker limit, converges to the FCI energy. With this method, FCI-quality calculations have been achieved on larger molecular systems ${ }^{44}$ and even periodic systems. ${ }^{42}$ F12 methods have also been combined with the FCIQMC technique ${ }^{44 \mid 45}$ as universal a posteriori corrections ${ }^{46[47}$ involving contractions of the the one- and two-body matrices with F12 integrals.

For periodic systems, plane waves are usually the most appropriate basis functions. However their smooth and non-local properties make the slow convergence in the description of electronic cusps an even more severe problem. This problem is tremendously enhanced in FCIQMC with large basis sets, aiming to reach the CBL. In this work we design an explicit correlation method to resolve this problem in such calculations. In FCIQMC, the matrix elements of the interaction operators (e.g., the two-body Coulomb operators, etc.) are used intensively and thus have to be either stored efficiently or calculated repeatedly on the fly. 
One advantage of the plane wave basis is that the two-body Coulomb matrix can be simply evaluated and need not be stored, alleviating the memory bottlenecks associated with storing the 4-index integrals of large systems. We would like to keep this advantage, so that the application of the intended explicit correlation method will not be limited to small systems. This requires that the involved effective potential and their matrix elements should be as simple as possible. In the next section, we will describe the new TC method designed for plane wave basis. In section 3, we will present our initial test calculations on three dimensional homogeneous electron gas models and, this will be followed by some conclusions and discussions in section 4 .

\section{Method}

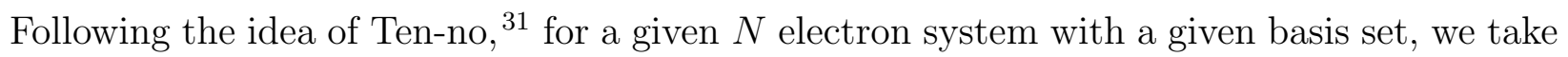
a fixed correlation factor $\tau$ in the Jastrow ansatz (2) and, try to determine the reference function $\Phi$ by solving approximately an eigenvalue equation

$$
\hat{H}_{\mathrm{TC}} \Phi=E \Phi
$$

where the effective Hamiltonian, the transcorrelated Hamiltonian, has a finite Baker-CampbellHausdorff expansion, upto a double commutator, owing to the fact that the correlation factor $\tau(\mathbf{R})$ is purely a function of the spatial coordinates of the electrons:

$$
\begin{aligned}
\hat{H}_{\mathrm{TC}} & \equiv e^{-\tau} \hat{H} e^{\tau} \\
& =\hat{H}+[\hat{H}, \tau]+\frac{1}{2}[[\hat{H}, \tau], \tau] \\
& =\hat{H}-\sum_{i}\left(\frac{1}{2} \nabla_{i}^{2} \tau+\left(\nabla_{i} \tau\right) \cdot \nabla_{i}+\frac{1}{2}\left(\nabla_{i} \tau\right)^{2}\right) .
\end{aligned}
$$

Here the TC Hamiltonian is non-Hermitian, owing to the presence of the single commutator term $[\hat{H}, \tau]$. For any eigenvalue of such operators, the corresponding left and right eigen- 
vectors are usually different. In solving this type of eigenvalue equations, approximations based on a variational treatment are usually troubled by the non-Hermitian nature of the matrix. Projection methods, however, such as the Power method provide a route forward

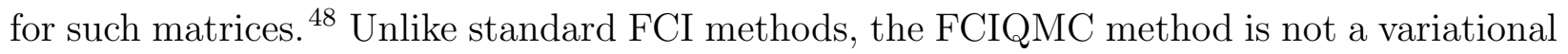
method but rather a stochastic version of the Power method. For the Jastrow ansatz, the time evolution of the wave function can be represented as

$$
\begin{aligned}
& \Psi(t)=e^{\tau} \Phi(t) \\
& \Phi(t)=e^{-t\left(\hat{H}_{\mathrm{TC}}-E_{0}\right)} \Phi(t=0),
\end{aligned}
$$

where equation (8) can be simply derived from equation (4)

$$
\begin{aligned}
\Phi(t) & =e^{-\tau} e^{-t\left(\hat{H}-E_{0}\right)} e^{\tau} \Phi(t=0) \\
& =\lim _{M \rightarrow \infty} e^{-\tau}\left(1-\frac{t\left(\hat{H}-E_{0}\right)}{M}\right)^{M} e^{\tau} \Phi(t=0) \\
& =\lim _{M \rightarrow \infty}\left(e^{-\tau}\left(1-\frac{t\left(\hat{H}-E_{0}\right)}{M}\right) e^{\tau}\right)^{M} \Phi(t=0) \\
& =\lim _{M \rightarrow \infty}\left(1-\frac{t\left(\hat{H}_{T C}-E_{0}\right)}{M}\right)^{M} \Phi(t=0) \\
& =e^{-t\left(\hat{H}_{\mathrm{TC}}-E_{0}\right)} \Phi(t=0)
\end{aligned}
$$

It is worth noticing that equation (8) is not constructed based on the eigenvalue equation (5), where one may get frustrated due to the non-Hermiticity and lack of variational bounds on $\hat{H}_{\mathrm{TC}}$. The equivalence of equation (4) and equation (8) reveals that methods based on these equations (such as FCIQMC) can handle properly the non-Hermiticity due to such kind of similarity transformations. Following equation (8), the FCIQMC method can be directly used for the TC Hamiltonian $\hat{H}_{\mathrm{TC}}$. The only difference is that here we are dealing with non-Hermitian operators, so that the involved matrix elements are non-symmetric. In calculations of these matrix elements, the operators should not be mixed up with their 
Hermitian conjugates (i.e., these operators should only be applied on to the right hand side).

For periodic systems, the orbital basis functions to be used are plane waves,

$$
\phi_{\mathbf{p}, \sigma}(\mathbf{r})=\frac{1}{\sqrt{\Omega_{0}}} e^{i \mathbf{p} \cdot \mathbf{r}},
$$

where $\Omega_{0}$ is the formal volume of the infinite system. All operators in the effective Hamiltonian can then be represented in terms of second quantisation. ${ }^{49}$ The fixed correlation factor, assumed to be spin-independent, can be expressed as

$$
\begin{aligned}
\tau & =\frac{1}{2} \sum_{i j} u_{0}\left(\mathbf{r}_{i}-\mathbf{r}_{j}\right) \\
& =\frac{1}{2} \sum_{\sigma \sigma^{\prime}} \sum_{\mathbf{k p q}}\left\langle\mathbf{p}-\mathbf{k}, \mathbf{q}+\mathbf{k}\left|u_{0}\right| \mathbf{p}, \mathbf{q}\right\rangle a_{\mathbf{p}-\mathbf{k}, \sigma}^{\dagger} a_{\mathbf{q}+\mathbf{k}, \sigma^{\prime}}^{\dagger} a_{\mathbf{q}, \sigma^{\prime}} a_{\mathbf{p}, \sigma} \\
& =\frac{1}{2 \Omega_{0}} \sum_{\sigma \sigma^{\prime}} \sum_{\mathbf{k p q}} \tilde{u}_{0}(\mathbf{k}) a_{\mathbf{p}-\mathbf{k}, \sigma}^{\dagger} a_{\mathbf{q}+\mathbf{k}, \sigma^{\prime}}^{\dagger} a_{\mathbf{q}, \sigma^{\prime}} a_{\mathbf{p}, \sigma},
\end{aligned}
$$

where $\tilde{u}_{0}(\mathbf{k})=\int e^{i \mathbf{k} \cdot \mathbf{r}} u_{0}(\mathbf{r}) d^{3} r$ is the Fourier transformation of $u_{0}$. Here the two-body correlation factor is assumed to be a function of $\mathbf{r}_{i j}=\mathbf{r}_{i}-\mathbf{r}_{j}$, due to translational symmetry. Similarly, for the other required two-body operators, we have the following expressions

$$
\begin{aligned}
\hat{W} & =\frac{1}{2 \Omega_{0}} \sum_{\sigma \sigma^{\prime}} \sum_{\mathbf{k p q}} \tilde{w}_{0}(\mathbf{k}) a_{\mathbf{p}-\mathbf{k}, \sigma}^{\dagger} a_{\mathbf{q}+\mathbf{k}, \sigma^{\prime}}^{\dagger} a_{\mathbf{q}, \sigma^{\prime}} a_{\mathbf{p}, \sigma}, \\
\frac{1}{2} \sum_{i} \nabla_{i}^{2} \tau & =-\frac{1}{2 \Omega_{0}} \sum_{\sigma \sigma^{\prime}} \sum_{\mathbf{k p q}} k^{2} \tilde{u}_{0}(\mathbf{k}) a_{\mathbf{p}-\mathbf{k}, \sigma}^{\dagger} a_{\mathbf{q}+\mathbf{k}, \sigma^{\prime}}^{\dagger} a_{\mathbf{q}, \sigma^{\prime}} a_{\mathbf{p}, \sigma}, \\
\sum_{i}\left(\nabla_{i} \tau\right) \nabla_{i} & =\frac{1}{2 \Omega_{0}} \sum_{\sigma \sigma^{\prime}} \sum_{\mathbf{k p q}}(\mathbf{p}-\mathbf{q}) \cdot \mathbf{k} \tilde{u}_{0}(\mathbf{k}) a_{\mathbf{p}-\mathbf{k}, \sigma}^{\dagger} a_{\mathbf{q}+\mathbf{k}, \sigma^{\prime}}^{\dagger} a_{\mathbf{q}, \sigma^{\prime}} a_{\mathbf{p}, \sigma},
\end{aligned}
$$

where $\hat{W}$ is the electronic (Coulomb) potential. The double commutator in equation $(6)$ is 
more complicated and gives rise to a three-body operator and a two-body operator

$$
\begin{aligned}
\frac{1}{2} \sum_{i}\left(\nabla_{i} \tau\right)^{2}= & \frac{1}{2} \sum_{i j k} \nabla_{i} u_{0}\left(\mathbf{r}_{i}-\mathbf{r}_{j}\right) \cdot \nabla_{i} u_{0}\left(\mathbf{r}_{i}-\mathbf{r}_{k}\right)+\frac{1}{2} \sum_{i j}\left(\nabla_{i} u_{0}\left(\mathbf{r}_{i}-\mathbf{r}_{j}\right)\right)^{2} \\
= & \frac{1}{2 \Omega_{0}^{2}} \sum_{\sigma \sigma^{\prime} \sigma^{\prime \prime}} \sum_{\mathbf{k} \mathbf{k}^{\prime} \mathbf{p q s}} \tilde{u}_{0}(\mathbf{k}) \tilde{u}_{0}\left(\mathbf{k}^{\prime}\right) \mathbf{k}^{\prime} \cdot \mathbf{k} a_{\mathbf{p}-\mathbf{k}, \sigma}^{\dagger} a_{\mathbf{q}+\mathbf{k}^{\prime}, \sigma^{\prime}}^{\dagger} a_{\mathbf{s}+\mathbf{k}-\mathbf{k}^{\prime}, \sigma^{\prime \prime}}^{\dagger} a_{\mathbf{s}, \sigma^{\prime \prime}} a_{\mathbf{q}, \sigma^{\prime}} a_{\mathbf{p}, \sigma} \\
& +\frac{1}{2 \Omega_{0}} \sum_{\sigma \sigma^{\prime}} \sum_{\mathbf{k p q}} \mathcal{F}\left(\left(\nabla u_{0}\right)^{2}\right)(\mathbf{k}) a_{\mathbf{p}-\mathbf{k}, \sigma}^{\dagger} a_{\mathbf{q}+\mathbf{k}, \sigma^{\prime}}^{\dagger} a_{\mathbf{q}, \sigma^{\prime}} a_{\mathbf{p}, \sigma}
\end{aligned}
$$

where $\mathcal{F}$ denotes Fourier transformation. A complete treatment of the three-body operator would be expensive, and we would like to treat it only approximately.

Periodic systems are usually treated by the supercell approach, $\frac{17}{17}$ where the infinite system is approximated by periodically arranged replicas of a finite cell $\Omega=L^{3}$. Due to this artificial periodic boundary condition, the $\mathbf{p}$ vector of the plane wave basis 10 is discretized (i.e., $\mathbf{p}=\frac{2 \pi}{L} \mathbf{n}, \mathbf{n} \in \mathcal{Z}^{3}$ ), and to make the basis finite we take a cutoff $|\mathbf{p}| \leq k_{c}$.

In the supercell approach, $u_{0}$ and $w_{0}$ have to fulfill the periodic boundary conditions. The periodic $w_{0}$ is usually constructed via periodic summation. ${ }^{17}$ Following the same idea, we construct the periodic correlation factor $u_{0}$ by applying the periodic summation on a local function $u$

$$
u_{0}(\mathbf{r})=\sum_{\mathbf{n} \in \mathcal{Z}^{3}} u(\mathbf{r}+\mathbf{n} L)
$$

However such summations are not practically needed if we work in k-space, since

$$
\frac{\tilde{u}_{0}(\mathbf{k})}{\Omega_{0}}=\frac{\tilde{u}(\mathbf{k})}{\Omega}, \quad \text { for } \mathbf{k}=\frac{2 \pi}{L} \mathbf{m}, \mathbf{m} \in \mathcal{Z}^{3}
$$

The only thing which needs to be taken care of is that the inverse Fourier transformation of $u_{0}$ does not exist, and instead, there is a Fourier series

$$
u_{0}\left(\mathbf{r}_{2}-\mathbf{r}_{1}\right)=\frac{1}{\Omega} \sum_{\mathbf{k}=2 \pi \mathbf{m} / L} \tilde{u}(\mathbf{k}) e^{i \mathbf{k} \cdot\left(\mathbf{r}_{2}-\mathbf{r}_{1}\right)}
$$


The two-body operator $\left(\nabla u_{0}\right)^{2}$ is very important for the short range correlation and, it is closely related to the infinite summation of all ladder diagrams in the linked cluster expansion. $\frac{19}{}$ By taking the periodic summation, $\left(\nabla u_{0}\right)^{2}$ can be expressed as

$$
\left(\nabla u_{0}\right)^{2}=-\frac{1}{\Omega^{2}} \sum_{\mathbf{k}, \mathbf{k}^{\prime}} \mathbf{k} \cdot \mathbf{k}^{\prime} \tilde{u}(\mathbf{k}) \tilde{u}\left(\mathbf{k}^{\prime}\right) e^{i \mathbf{k} \cdot\left(\mathbf{r}_{2}-\mathbf{r}_{1}\right)} e^{i \mathbf{k}^{\prime} \cdot\left(\mathbf{r}_{2}-\mathbf{r}_{1}\right)}
$$

whose Fourier transformation is now

$$
\mathcal{F}\left(\left(\nabla u_{0}\right)^{2}\right)(\mathbf{k})=-\frac{\Omega_{0}}{\Omega}\left(\frac{1}{\Omega} \sum_{\mathbf{k}^{\prime}}\left(\mathbf{k}-\mathbf{k}^{\prime}\right) \cdot \mathbf{k}^{\prime} \tilde{u}\left(\mathbf{k}-\mathbf{k}^{\prime}\right) \tilde{u}\left(\mathbf{k}^{\prime}\right)\right)
$$

As explained before, the correlation factor $\tau$ is designed here mainly to capture the short range cusp. In the short range limit, we have the asymptotic solution $\underline{49}$

$$
\tilde{u}(\mathbf{k})=-\frac{4 \pi}{\mathbf{k}^{4}}, \text { when } k \sim \infty
$$

which is the cusp condition expressed in the k-space. This expression is derived for unlike spin pairs, while for electron pairs with the same spin $\tilde{u}(\mathbf{k}) \sim-\frac{2 \pi}{\mathbf{k}^{4}}$. In principle we can use any kind of local function $u$ in the TC calculation, as long as it satisfies the cusp condition. However, since we intend to introduce approximations in the treatment of the three-body term, we want $u$ to be small and to vanish in the CBL. Therefore we design the following correlation factor

$$
\tilde{u}(\mathbf{k})= \begin{cases}-\frac{4 \pi}{k^{4}}, & |\mathbf{k}|>k_{c} \\ 0, & |\mathbf{k}| \leq k_{c}\end{cases}
$$

where $k_{c}$ is the cutoff parameter of the basis set. The idea behind this construction is simple: since the wave function can already be described by a configuration description up to the given level of resolution (characterized by $k_{c}$ ), it is only needed to be improved in the finer region of resolution by means of the correlation factor. In real space the above correlation 
factor becomes:

$$
u(r)=-\frac{r}{\pi}\left(\operatorname{si}\left(k_{c} r\right)+\frac{\cos \left(k_{c} r\right)}{k_{c} r}+\frac{\sin \left(k_{c} r\right)}{\left(k_{c} r\right)^{2}}\right),
$$

where $\operatorname{si}(x)=-\int_{x}^{\infty} \frac{\sin x}{x} d x$ is the sine integral. A sketch of $u(r)$ is presented in Figure 1 . which looks like a small "hole" with the depth $=-2 / \pi k_{c}$ and the width $\sim \pi / k_{c}$. Taylor expansion of $u$ in the small $r$ region can be calculated for the leading terms

$$
u(r)=-\frac{2}{\pi k_{c}}+\frac{r}{2}+\cdots,
$$

where the second term satisfy the cusp condition for unlike spin pairs. In the large $r$ region, the magnitude of $u(r)$ decades like $1 / r^{2}$.

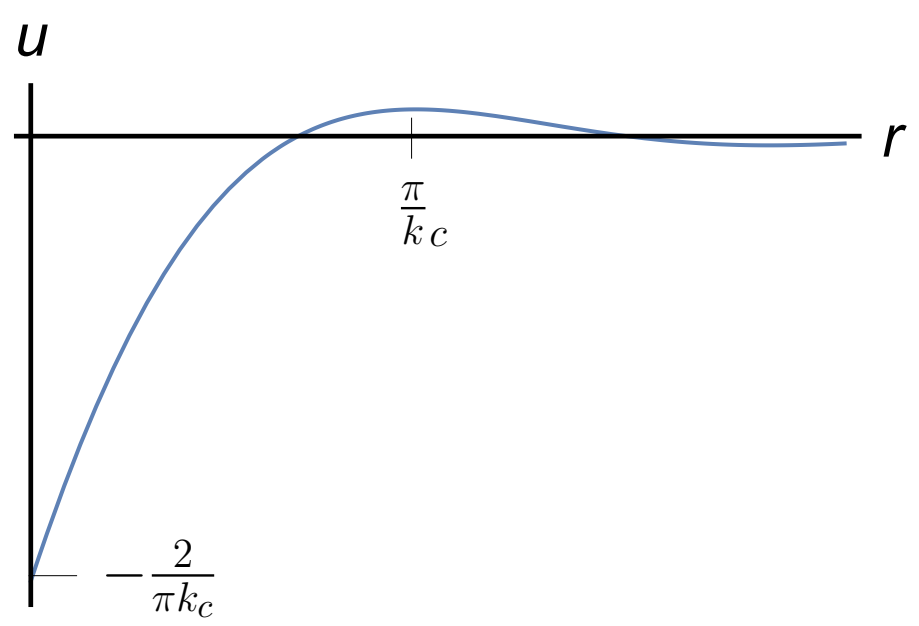

Figure 1: A sketch of the correlation factor $u(r)$.

In principle, we can also use a spin-dependent correlation factor, namely to reduce the correlation factor for parallel spin pairs by one half. However, the existence of exchange "holes" between parallel-spin electrons keeps them largely apart anyway, and as a consequence the system energy is not very sensitive to the cusp between such pairs. Therefore the use of spin-dependent correlation factors does not significantly improve the convergence rate of energy. Rather, spin-dependent Jastrow factors induce undesirable spin contamination $\sqrt{50}$ into the wave function, so that the wave function can not be an eigenstate of $S^{2}$.

With the above short ranged correlation factor, we can largely ignore the complicated 
three-body operator in equation (15) and take only a simple RPA type contribution from it. This contribution is represented by a two-body operator

$$
\frac{N-2}{2 \Omega^{2}} \sum_{\sigma \sigma^{\prime}} \sum_{\mathbf{k p q}} k^{2} \tilde{u}^{2}(\mathbf{k}) a_{\mathbf{p}-\mathbf{k}, \sigma}^{\dagger} a_{\mathbf{q}+\mathbf{k}, \sigma^{\prime}}^{\dagger} a_{\mathbf{q}, \sigma^{\prime}} a_{\mathbf{p}, \sigma}
$$

which is generated by a contraction of the $a_{\mathbf{s}+\mathbf{k}-\mathbf{k}^{\prime}, \sigma^{\prime \prime}}^{\dagger} a_{\mathbf{s}, \sigma^{\prime \prime}}$ pairs in equation 15 (i.e., by a summation of those terms where $\mathbf{k}=\mathbf{k}^{\prime}$ ). This term makes the dominant contribution of the three-body operator to the long range correlation, and is closely related to the summation of all ring diagrams in the linked cluster expansion. $.51+53$ In the current method, the correlation factor is short ranged and, therefore the contribution of these terms will be very small. In the applications studied in this paper, namely the homogeneous electron gas in the $r_{s}$ range from 0.5 to 5 , this contribution was less than $1 \%$ of the total correlation energy. We nevertheless keep it in the method for two reasons: firstly it partly recovers the three-body contributions and, secondly it can be used to estimate the magnitude of error due to the missing threebody terms. Last but not least, the use of this term incurs almost no extra computational cost.

Putting all terms together, we have the following two-body effective potential

$$
\begin{aligned}
\hat{W}_{\mathrm{eff}}= & \frac{1}{2 \Omega} \sum_{\sigma \sigma^{\prime}} \sum_{\mathbf{k p q}} \tilde{w}_{\mathrm{eff}}(\mathbf{k}, \mathbf{p}, \mathbf{q}) a_{\mathbf{p}-\mathbf{k}, \sigma}^{\dagger} a_{\mathbf{q}+\mathbf{k}, \sigma^{\prime}}^{\dagger} a_{\mathbf{q}, \sigma^{\prime}} a_{\mathbf{p}, \sigma} \\
\tilde{w}_{\mathrm{eff}}(\mathbf{k}, \mathbf{p}, \mathbf{q})= & \tilde{w}(\mathbf{k})+k^{2} \tilde{u}(k)-(\mathbf{p}-\mathbf{q}) \cdot \mathbf{k} \tilde{u}(\mathbf{k})-\frac{N_{e}-2}{\Omega} k^{2} \tilde{u}^{2}(\mathbf{k}) \\
& +\frac{1}{\Omega} \sum_{\mathbf{k}^{\prime}}\left(\mathbf{k}-\mathbf{k}^{\prime}\right) \cdot \mathbf{k}^{\prime} \tilde{u}\left(\mathbf{k}-\mathbf{k}^{\prime}\right) \tilde{u}\left(\mathbf{k}^{\prime}\right)
\end{aligned}
$$

where $\tilde{w}(\mathbf{k})$ is the original Coulomb potential

$$
\tilde{w}(\mathbf{k})= \begin{cases}\frac{4 \pi}{k^{2}}, & \mathbf{k} \neq \mathbf{0}, \\ 0, & \mathbf{k}=\mathbf{0} .\end{cases}
$$


In equation (27), the last term coming from $\mathcal{F}\left((\nabla u)^{2}\right)(\mathbf{k})$ is only a function of $\mathbf{k}$ and can be easily prepared and stored before the FCIQMC simulation. Unlike other terms, this term is non-zero at $\mathbf{k}=\mathbf{0}$. Practical implementation of this TC method in FCIQMC calculations is straightforward. We need only to replace the pure electronic Coulomb potential $\tilde{w}$ with the TC effective potential $\tilde{w}_{\text {eff }}$, and this requires only a very small modification of the existing code. Since calculation of the Coulomb potential makes up only a very small portion of the total computational cost of the FCIQMC method, use of the effective potential will not make the computations more expensive.

\section{Results}

Homogeneous electron gases (HEG) are important models for the investigation of electron correlation in solids. They also play a fundamental role in the development of density functional theory (DFT). ${ }^{54}$ These models have been intensively studied by variational and diffusion quantum Monte Carlo simulations. $\frac{[55-59}{56}$ The DMC method is very efficient and can be applied to fairly large systems. On the other hand this method does not solve the problem completely, owing to the fixed-node approximation, even though this can be reduced by means of back-flow and multi-determinant techniques. The FCIQMC method contains in principle no bias, and offers a way to investigate the fixed node error. Recently the FCIQMC method has been used to investigate the three dimensional HEG model ${ }^{60}[62$ Due to the cusp singularity of the wave functions, the calculation suffers from slow convergence with respect to the basis size, with the error of the calculated energy being proportional to $M^{-1}, M$ being the total number of basis functions. Extrapolations based on this $M^{-1}$ behavior are used to estimate the results in the CBL. For an accurate result, calculations need to be performed on fairly large basis sets in order to reach the $M^{-1}$ regime, and the computational cost increases sharply with basis size.

In order to study the efficiency of the new TC method, we have performed calculations 
on the same 3D HEG systems as investigated in the previous studies. $\frac{60[61}{6 w o}$ different supercell sizes are used, containing 14 and 54 electrons respectively. For the 14 electron cell, calculations are carried out on four different densities with Wigner-Seitz radius $r_{s}=0.5$, 1.0, 2.0 and 5.0 respectively. For the 54 electron cell, we can only get converged results for $r_{s}=0.5$ and 1.0 , since the required total number of walkers increases rapidly with $r_{s}$. We use the initiator-FCIQMC ( $i$-FCIQMC) method with the initiator parameter set to be 3.0. In addition we used the semi-stochastic method ${ }^{63}$ using the $|D|=10000$ leading determinants in the deterministic space ${ }^{64}$ as implemented in the NECI code $\underline{65}$
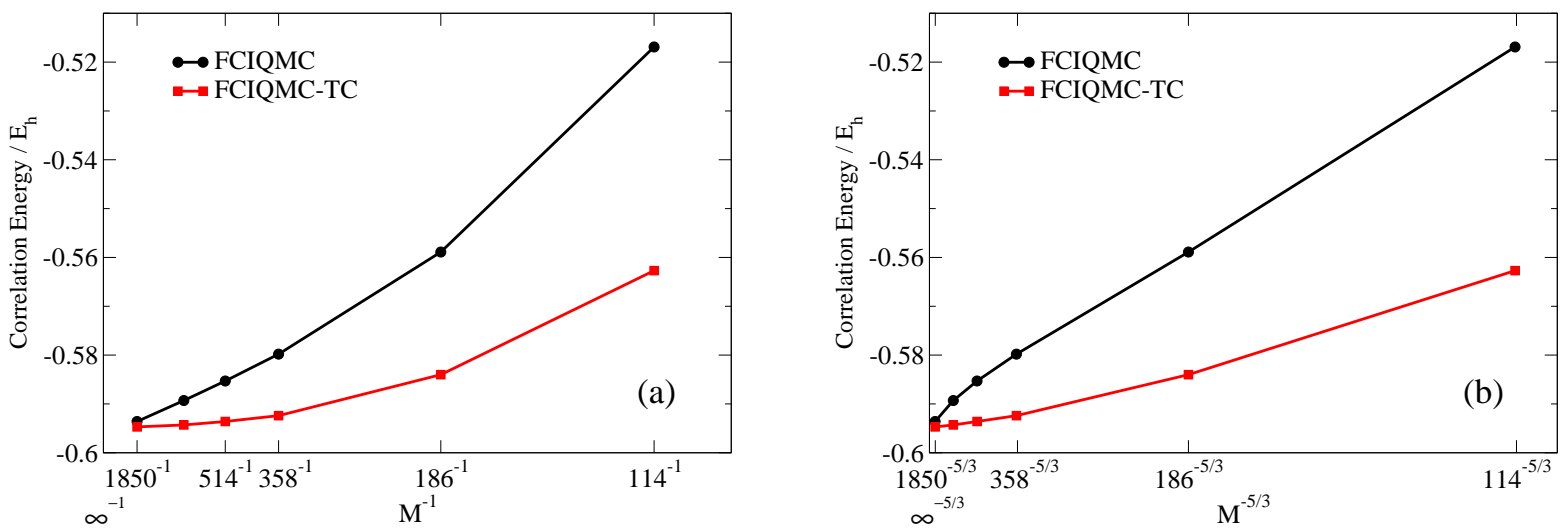

Figure 2: (a) Total correlation energy as a function of $M^{-1}$ calculated by FCIQMC and FCIQMC-TC methods for the 14 electron system with $r_{s}=0.5$. (b) The same results but presented as a function of $M^{-5 / 3}$.

In Figure 2(a), the total correlation energy as a function of $M^{-1}$ is presented for the 14 electron system with $r_{s}=0.5$, where the two different results are calculated by FCIQMC method with the effective TC Hamiltonian (FCIQMC-TC) and the original FCIQMC method respectively. Here $M$, the number of spin orbital basis functions, is chosen to be 114, 186, 358, 514, 778 and 1850 respectively. The FCIQMC result shows an asymptotic linear convergence with respect to $M^{-1}$, while the result of FCIQMC-TC has a higher order of convergence. According to the theoretical analysis in Appendix A, the best asymptotic convergence we can expect is $M^{-5 / 3}$. We also present the same results in Figure 2(b) as functions of $M^{-5 / 3}$. 
The result of FCIQMC-TC shows a roughly linear behavior in the large $M$ region, and in the small $M$ region the convergence is faster. The asymptotic $M^{-5 / 3}$ convergence behavior offers a possibility of extrapolations to the CBL, in case of need. For the result in Figure 2. such an extrapolation is not necessary, since $1850^{-5 / 3}$ is already very close to the origin $\left(\infty^{-5 / 3}\right)$ and the FCIQMC-TC result has already converged at 0.1 milihartree $\left(m E_{h}\right)$ level.
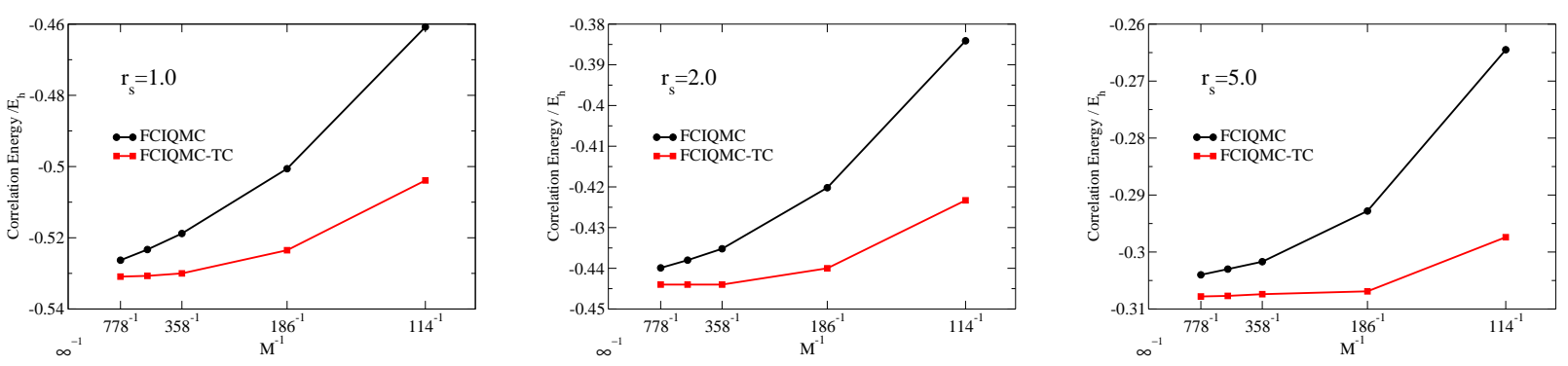

Figure 3: Total correlation energy as a function of $M^{-1}$ calculated by FCIQMC and FCIQMC-TC methods for the 14 electron system with $r_{s}=1.0,2.0$ and 5.0 respectively.

Similar behaviors are observed for other densities of the 14 electron system. In Figure 3 , the convergence with respect to $M^{-1}$ of the two different calculations are presented for $r_{s}=1.0,2.0$ and 5.0 respectively in three plots. It turns out that for larger $r_{s}$ the result converges faster. For $r_{s}=1.0$ the FCIQMC-TC energy has converged within a milihartree error at $M=514$, while for $r_{s}=2.0$ and 5.0 such a convergence can already been reached at $M=358$ and 186 respectively. However, this does not mean that simulations for larger $r_{s}$ s are easier, since the required number of walkers $\left(N_{w}\right)$ increases sharply with $r_{s}$, in order to reduce the initiator error. For $r_{s}=0.5 \sim 2.0$, calculations are performed mostly with $N_{w}=10^{7} \sim 10^{8}$, while for $r_{s}=5.0$ we need $N_{w}=10^{9}$ already at $M=114 \sim 358$ and even $N_{w}=10^{10}$ at $M=514$ and 778. For such systems, in order to demonstrate the $M^{-5 / 3}$ asymptotic convergence we have to deal with larger basis sets, which will require even larger $N_{w}$ 's, but on the other hand such difficult calculations are not needed for our accuracy requirement.

Computations on the 54-electron systems are much more expensive and with present computational resources we can get converged results only for $r_{s}=0.5$ and $r_{s}=1.0$. For 

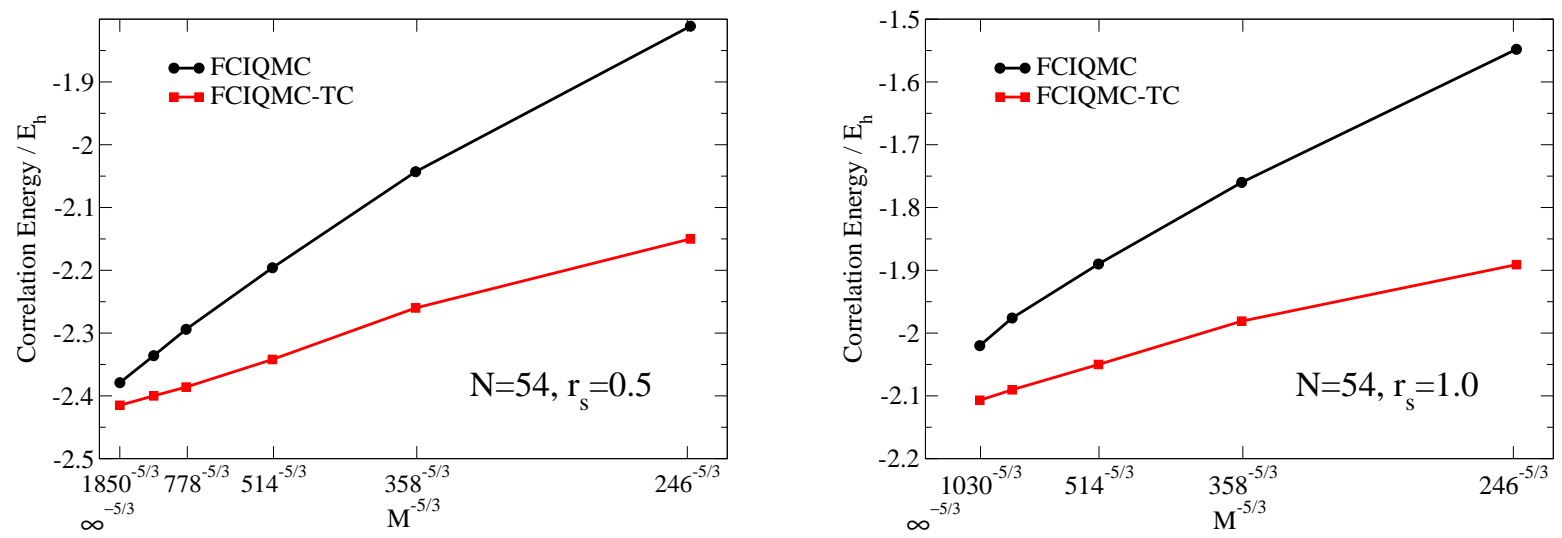

Figure 4: Total correlation energy as a function of $M^{-5 / 3}$ calculated by FCIQMC and FCIQMC-TC methods for the 54 electron systems with $r_{s}=0.5$ and $r_{s}=1.0$.

$r_{s}=0.5$, calculations are performed with 6 different basis sets with $M=246 \rightarrow 1850$, where $N_{w}=10^{8}$ is found to be sufficient for all of them. For $r_{s}=1.0$, we can get converged results for $M$ up to 1030 . For $M=246,358$ and 514, the number of walkers $N_{w}$ is taken to be $10^{10}$, while for $M=778$ and 1030 we need $N_{w}=2 \times 10^{10}$. In Figure 4 , the results for 54-electron systems are presented as functions of $M^{-5 / 3}$ for $r_{s}=0.5$ and $r_{s}=1.0$. The asymptotic behaviors are clearer than those of the 14-electron systems for the FCIQMC-TC results and extrapolations are used to evaluate the results in the CBL.

Table 1: Total correlation energies in the complete basis limit for a variety of $N$ and $r_{s}$ calculated with FCIQMC-TC method. Extrapolation based on $M^{-5 / 3}$ behavior is used for the 54-electron systems. The results are compared with the previous FCIQMC results $\frac{6162}{62}$ and the back-flow DMC results. $\frac{58}{}$

\begin{tabular}{|c|c|c|c|c|c|}
\hline \multirow{2}{*}{$\begin{array}{c}r_{s} \\
\text { (a.u.) }\end{array}$} & \multirow[b]{2}{*}{$\mathrm{N}$} & \multicolumn{4}{|c|}{$E_{\text {corr }}$ (a.u.) } \\
\hline & & FCIQMC-TC & FCIQMC & FCIQMC 62 & BF-DMC \\
\hline \multirow[t]{2}{*}{0.5} & 14 & $-0.5948(2)$ & $-0.5959(7)$ & $-0.59467(9)$ & \\
\hline & 54 & $-2.425(1)$ & $-2.435(7)$ & & $-2.387(2)$ \\
\hline \multirow[t]{2}{*}{1.0} & 14 & $-0.5309(2)$ & $-0.5316(4)$ & $-0.5313(2)$ & \\
\hline & 54 & $-2.134(2)$ & $-2.124(3)$ & & $-2.125(2)$ \\
\hline 2.0 & 14 & $-0.4440(3)$ & $-0.444(1)$ & & \\
\hline 5.0 & 14 & $-0.3078(3)$ & $-0.307(1)$ & & \\
\hline
\end{tabular}


In Table 1 the complete basis limit results are presented for all calculated systems. The results for the 14-electron systems are simply taken from those of the used largest basis sets. Within the given error bars, these results are already converged to the CBL. The results for $N=54$ are obtained by extrapolations based on the $M^{-5 / 3}$ convergence rate. Compared with the previous FCIQMC results, the new results agree well for the 14-electron systems, with the differences in total energies $\leq 1 m E_{h}$. The new results are also in good agreement with the recent high-order Coupled-Cluster study of the $N=14$ electron system by Neufeld and Thom. ${ }^{62}$ The small differences between the FCIQMC results of Shepherd et al. .61 and Neufeld and Thom ${ }^{62}$ for the 14-electron systems arise because of the use of different extrapolation formulae to the infinite-basis set limit, the former being based on $M^{-1}$ whilst the latter includes higher order terms $\left(b_{0}+b_{1} M^{-1}+b_{2} M^{-2)}\right.$. This allows the use of a larger number of points in the extrapolation procedure (with smaller $M$ ), and leads to somewhat higher extrapolated correlation energies, the implication being that a simple $M^{-1}$ extrapolation tends to over-shoot the exact result unless a sufficiently large $M$ has been reached. Although the differences are not large, this indicates that the precise form of extrapolation is indeed consequential, and provides a further motivation to try to minimize basis-set errors through analytic means as far as possible.

For $N=54$, the new transcorrelated result at $r_{s}=0.5$ is $10 m E_{h}$ above the previous FCIQMC result of Shepherd et al.$\underline{60}$ based on a $M^{-1}$ extrapolation. At this density, the FCIQMC-TC result is about $40 m E_{h}$ below the back-flow DMC result $\frac{58}{51}$ and this indicates that the fixed-node error of the BF-DMC result is still quite large for $r_{s}=0.5$. For $r_{s}=1.0$, the previous FCIQMC result for $N=54$ is roughly the same as the BF-DMC result, while the new result is about $10 m E_{h}$ below the BF-DMC result. This reveals the fixed nodes error decays very rapidly with $r_{s}$ and, we expect that, at even larger $r_{s}$ the fixed-node error of BF-DMC will be even smaller and hence can be ignored.

The effective TC potential in equation (27), now denoting as $\tilde{w}_{\text {eff }}(\mathbf{k}, \mathbf{p}, \mathbf{q})=\tilde{w}(\mathbf{k})+$ $\tilde{w}_{\mathrm{TC}}[\tilde{u}](\mathbf{k}, \mathbf{p}, \mathbf{q})$, is constructed with $\tilde{u}(\mathbf{k})$ defined in equation $(22)$. Since $\tilde{u}(\mathbf{k})$ contains only 


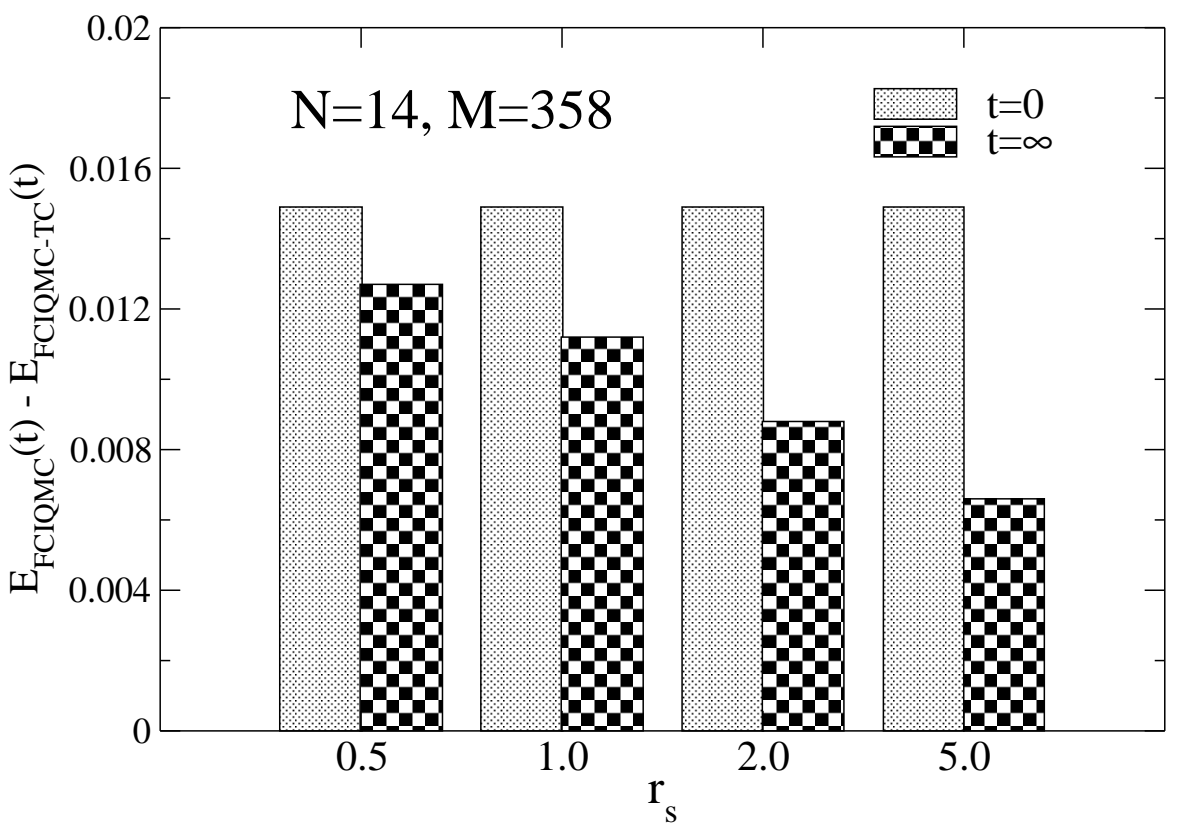

Figure 5: Energy differences $\Delta E(t)$ at $t=0$ and $t=\infty$ for 14-electron systems with $M=358$ presented for all different densities $r_{s}=0.5 \sim 5.0$.

high frequency terms, it might be expected that the effective potential $\tilde{w}_{\mathrm{TC}}$ has a weak coupling to the low frequency basis space and thus this potential behaves roughly like a constant potential in the dynamic evolution. If this would be the case, it should be expected that the energy difference produced by $\tilde{w}_{\mathrm{TC}}$

$$
\Delta E(t)=E_{\mathrm{FCIQMC}}(t)-E_{\mathrm{FCIQMC}-\mathrm{TC}}(t),
$$

should be approximately independent of imaginary time. In Figure 5, such energy differences for the 14-electron systems with $r_{s}=0.5 \sim 5.0$ and on the basis $M=358$ is presented for $t=0$ and $t=\infty$. In the calculation, the initial wave function are always chosen as the Hartree-Fock (HF) wave function. It can be seen that at high density $\Delta E(0)$ is close to $\Delta E(\infty)$, but at low density they are quite different. This indicates that the effective potential $\tilde{w}_{\mathrm{TC}}$ does have a coupling to the wave function, especially at low density region. 
It turns out that, for a given number of particles $N$ and a given basis size $M, \Delta E(0)$ is independent of $r_{s}$. This can be easily understood by scaling arguments. At HF level, the kinetic energy $\propto r_{s}^{-2}$ and the exchange energy $\propto r_{s}^{-1}$, because the corresponding operators $-\frac{1}{2} \sum_{i} \nabla_{i}^{2}$ and $\sum_{i j} 1 / r_{i j}$ scale like $r_{s}^{-2}$ and $r_{s}^{-1}$ respectively. By referring to equation 6, we get the expression of $\Delta E(0)$

$$
\Delta E(0)=\left\langle\Phi_{\mathrm{HF}}\left|\frac{1}{2} \sum_{i}\left(\nabla_{i} \tau\right)^{2}\right| \Phi_{\mathrm{HF}}\right\rangle
$$

Based on equation (11) and equation (23), it is not difficult to find that $\sum_{i}\left(\nabla_{i} \tau\right)^{2}$ scales as a constant of $r_{s}$ and, therefore $\Delta E(0)$ does not depend on $r_{s}$.

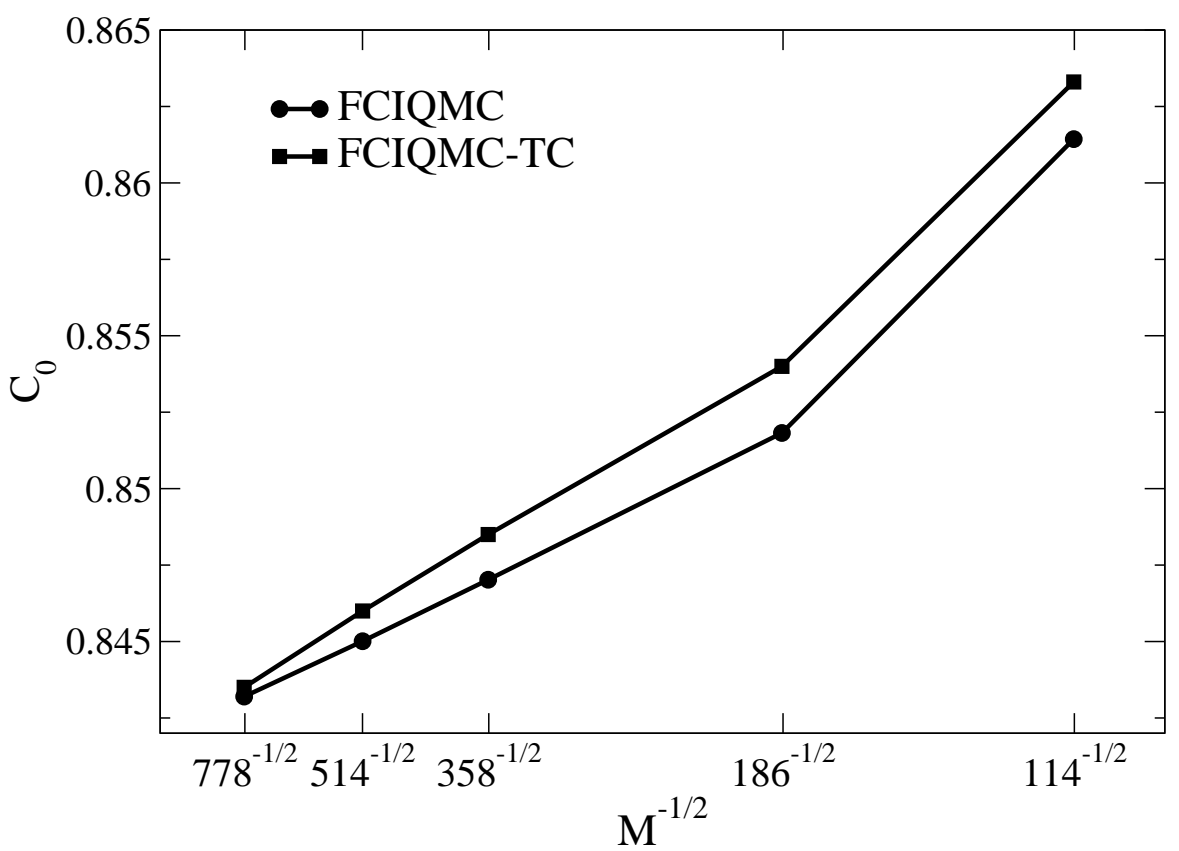

Figure 6: CI coefficient of the Hartree-Fock determinant in the final solutions of wave function with the two different methods presented for different basis sets. The results is calculated for the 14-electron system with $r_{s}=2.0$.

The conclusion that the effective potential $\tilde{w}_{\text {TC }}$ couples to the wave function can also be verified by looking at the difference of the final solutions of wave function between the two 
different methods. In Figure 6, the CI coefficient of the Hartree-Fock determinant $\left(C_{0}\right)$ in the final solutions of wave function with the two different methods is presented for different basis sets. The results is calculated for the 14-electron system with $r_{s}=2.0$. $C_{0}$ of the FCIQMC-TC result is found clearly larger than that of the FCIQMC result. This also serves as an evidence that the effective potential does couples to the wave function space. In the Figure, it can be found that both curves show a rough $1 / \sqrt{M}$ convergence. For the FCIQMC result, this can be easily understood based on variational argument, that since the energy converges like $M^{-1}$, the wave function should converge like $1 / \sqrt{M}$. As for the FCIQMC-TC result, where the energy converges like $M^{-5 / 3}$, the variational argument can not be applied, since the Hamiltonian is not fixed and itself depends on the basis.

\section{Conclusions}

In this work, we have designed a simple but efficient transcorrelated method for plane wave basis functions. The effective Hamiltonian contains only several two-body operators and thus can be easily implemented. In order to systematically reduce the error due to the neglect of the three-body operator in the original transcorrelated Hamiltonian, the correlation factor is constructed in a natural and systematic way according to the basis set. As an initial test, this simple effective Hamiltonian is used in FCIQMC calculations of homogeneous electron gas models. The results demonstrate that, with the same computational cost, this simple method can improve the FCIQMC convergence rate from $O\left(M^{-1}\right)$ to $O\left(M^{-5 / 3}\right)$.

We have also demonstrated that the effective transcorrelated Hamiltonian does couple

to the wave function and changes the dynamic evolution of the FCIQMC simulations. This means that the FCIQMC-TC results can not be precisely estimated based on an a posteriori use of the effective Hamiltonian.

The effective Hamiltonian, in principle, can also be applied to other projection methods such as the coupled cluster method. Due to the simple structure of the effective Hamiltonian, 
its implementation should be easier than usual F12 methods. We are currently working on this implementation.

Generalization of this method to other type of basis is straightforward and the basis dependent correlation factors can be constructed as follows: take first a usual F12 factor, for example a Slater type geminal $f\left(r_{12}\right)=\exp \left(-\gamma r_{12}\right)$, then the correlation factor can be constructed by projection against the current (orthonormal) basis set $\left\{\phi_{i}, i=1, \cdots, M\right\}$

$$
\begin{aligned}
u\left(\mathbf{r}_{1}, \mathbf{r}_{2}\right) & =f\left(\left|\mathbf{r}_{1}-\mathbf{r}_{2}\right|\right)-\sum_{i, j}^{M} F_{i j} \phi_{i}\left(\mathbf{r}_{1}\right) \phi_{j}\left(\mathbf{r}_{2}\right), \\
F_{i j} & =\int f\left(\left|\mathbf{r}_{1}-\mathbf{r}_{2}\right|\right) \phi_{i}\left(\mathbf{r}_{1}\right) \phi_{j}\left(\mathbf{r}_{2}\right) d^{3} r_{1} d^{3} r_{2} .
\end{aligned}
$$

The effective two-body transcorrelated potential can then be calculated with this correlation factor. Comparing with the plane wave basis, the expressions may become redundant. Therefore the induced one-body and two-body matrix elements have to be prepared and stored. We plan to work on this in the near future.

\section{Acknowledgments}

We thank A. Grüneis, J. Brand, P. Jeszenszki and D. Tew for helpful discussions. Calculations are partly carried out on Hydra clusters in Supercomputing Center Garching. 


\section{Appendix}

\section{A Regularities and Convergence Rates}

The regularity of many body wave functions $\Psi$ is $C^{0,1}$ and the non-smoothness comes largely from the cusps between electron pairs. (In the present analysis we concentrate only on the electronic cusps, and ignore the electron-nuclear cusps.) For a understanding of the relation between the regularity and the convergence rate, it is enough to take the example of a two electron system, which is essentially a one body problem in the center of mass coordinate. Due to the electronic cusp, the short range behavior of the exact wave function for a three dimensional non spin-polarised system looks like $|\tilde{\psi}(\mathbf{k})| \propto k^{-4}$, for $k \rightarrow \infty$. This means that for a finite plane wave basis with a cutoff at $k_{c}$, the error of wave function due to the missing resolution for the cusp can be estimated as

$$
\delta \tilde{\psi}(\mathbf{k}) \propto \begin{cases}\frac{1}{k^{4}}, & |\mathbf{k}|>k_{c} \\ 0, & |\mathbf{k}| \leq k_{c}\end{cases}
$$

The error of a variational energy can then be approximated as (ignoring any change of normalisation):

$$
\begin{aligned}
\delta E \approx\langle\delta \psi|\hat{H}| \delta \psi\rangle & \\
& \approx \quad \frac{1}{2} \int \delta \tilde{\psi}(\mathbf{k}) k^{2} \delta \tilde{\psi}(\mathbf{k}) d^{3} k \\
& \propto \quad \frac{1}{k_{c}^{3}},
\end{aligned}
$$

where we have used that fact that the leading contribution comes from the kinetic energy. By using a Jastrow factor, the regularity of the wave function is improved to $C^{1,1}$, which means that now the first order derivatives of $\psi$ is in $C^{0,1}$. This leads to an asymptotic short range behavior of the gradient of the reference function $|\widetilde{\nabla \phi}(\mathbf{k})| \propto k^{-4}$, for $k \rightarrow \infty$. Similarly, the 
energy error for a finite basis set can be estimated as

$$
\begin{aligned}
\delta E & \approx \frac{1}{2} \int|\delta \widetilde{\nabla \phi}(\mathbf{k})|^{2} d^{3} k \\
& \propto \frac{1}{k_{c}^{5}}
\end{aligned}
$$

Since the basis size $M \propto k_{c}^{3}$, we see that the Jastrow factor improves the convergence rate from $\mathcal{O}\left(M^{-1}\right)$ to $\mathcal{O}\left(M^{-5 / 3}\right)$.

\section{References}

(1) Helgaker, T.; Jørgensen, P.; Olsen, J. Molecular Electronic-Structure Theory; Wiley: New York, 1999.

(2) Kato, T. On the Eigenfunctions of Many-Particle Systems in Quantum Mechanics. Commun. Pure Appl. Math. 1957, 10, 151-177.

(3) Kutzelnigg, W. $r_{12}$-Dependent terms in the wave function as closed sums of partial wave amplitudes for large 1. Theoret. Chim. Acta 1985, 68, 445-469.

(4) Kutzelnigg, W.; Klopper, W. Wave functions with terms linear in the interelectronic coordinates to take care of the correlation cusp. I. General theory. J. Chem. Phys. 1991, 94, 1985-2001.

(5) Klopper, W.; Manby, F. R.; Ten-no, S.; Valeev, E. F. R12 methods in explicitly correlated molecular electronic structure theory. Int. Rev. in Phys. Chem. 2006, 25, 427-468.

(6) Hättig, C.; Klopper, W.; Köhn, A.; Tew, D. Explicitly correlated electrons in molecules. Chem. Rev. 2012, 112, 4-47.

(7) Tew, D.; Hättig, C.; Bachorz, R. A.; Klopper, W. Recent Progress in Coupled Cluster 
Methods - Theory and Applications. Dordrecht/Heidelberg/London/New York, 2010; pp 535-572.

(8) Werner, H.-J.; Adler, T. B.; Knizia, G.; Manby, F. R. Recent Progress in Coupled Cluster Methods - Theory and Applications. Dordrecht/Heidelberg/London/New York, 2010; pp 573-620.

(9) Kong, L.; Bischoff, F. A.; Valeev, E. F. Explicitly Correlated R12/F12 Methods for Electronic Structure. Chem. Rev. 2012, 112, 75-107.

(10) Valeev, E. F. Improving on the resolution of the identity in linear R12 ab initio theories. Chem. Phys. Lett. 2004, 395, 190-195.

(11) Jastrow, R. Many-body problems with strong forces. Phys. Rev. 1955, 98, 1479-1484.

(12) Fournais, S.; Hoffmann-Ostenhof, M.; Hoffmann-Ostenhof, T.; Østergaard Sørensen, T. Sharp Regularity Results for Coulombic Many-Electron Wave Functions. Commun. Math. Phys. 2005, 255, 183-227.

(13) Fournais, S.; Hoffmann-Ostenhof, M.; Hoffmann-Ostenhof, T.; Østergaard Sørensen, T. Analytic Structure of Many-Body Coulombic Wave Functions. Commun. Math. Phys. 2009, 289, 291-310.

(14) Kutzelnigg, W.; Morgen, J. D. Rates of convergence of the partial-wave expansions of atomic correlation energies. J. Chem. Phys. 1992, 96, 4484-4508.

(15) Ceperley, D. Ground state of the fermion one-component plasma: A Monte Carlo study in two and three dimensions. Phys. Rev. 1978, 18, 3126-3138.

(16) Umrigar, C. J.; Nightingale, M. P.; Runge, K. J. A diffusion Monte-Carlo algorithm with very small time-step errors. J. Chem. Phys. 1993, 99, 2865-2890.

(17) Foulkes, W. M. C.; Mitas, L.; Needs, R. J.; Rajagopal, G. Quantum Monte Carlo simulations of solids. Rev. Mod. Phys. 2001, 73, 33-83. 
(18) Clark, J. W.; Westhaus, P. Cluster expansions in many-fermion theory. I. " Factorcluster" formalisms. J. Math. Phys. 1968, 9, 131-148.

(19) Talman, J. Linked-cluster expansion for Jastrow-type wave functions and its application to the electron-gas problem. Phys. Rev 1974, A 10, 1333-1344.

(20) Gaudoin, R.; Nekovee, M.; Foulkes, W. M. C.; Needs, R. J.; Rajagopal, G. Inhomogeneous random-phase approximation and many-electron trial wave function. Phys. Rev. 2001, B 63, 115115.

(21) Krotscheck, E. Variations on the electron-gas. Ann. Phys. (N.Y.) 1984, 155, 1-55.

(22) Krotscheck, E. Theory of inhomogeneous quantum seytems. III. Variational wave functions for Fermi fluids. Phys. Rev. 1985, B 31, 4267-4278.

(23) Boys, S. F. Some Bilinear Convergence Characteristics of the Solutions of Dissymmetric Secular Equations. Proc. Roy. Soc. 1969, A 309, 195-208.

(24) Boys, S. F.; Handy, N. C. A Condition to Remove the Indeterminacy in Interelectronic Correlation Functions. Proc. Roy. Soc. 1969, A 309, 209-220.

(25) Boys, S. F.; Handy, N. C. The Determination of Energies and Wavefunctions with Full Electronic Correlation. Proc. Roy. Soc. 1969, A 310, 43-61.

(26) Boys, S. F.; Handy, N. C. A Calculation for the Energies and Wavefunctions for States of Neon with Full Electronic Correlation Accuracy. Proc. Roy. Soc. 1969, A 310, 63-78.

(27) Boys, S. F.; Handy, N. C. A First Solution, for LiH, of a Molecular Transcorrelated Wave Equation by Means of Restricted Numerical Integration. Proc. Roy. Soc. 1969, A 311, 309-329.

(28) Handy, N. C. Energies and expectation values for Be by transcorrelated method. J. Chem. Phys. 1969, 51, 3205-3212. 
(29) Handy, N. C. The transcorrelated method for accurate correlation energies using gaussian-type functions. Mol. Phys. 1972, 23, 1-27.

(30) Handy, N. C. Towards and understanding of the form of correlated wavefunctions for atoms. J. Chem. Phys. 1973, 58, 279-287.

(31) Ten-no, S. A feasible transcorrelated method for treating electronic cusps using a fronzen Gaussina geminal. Chem. Phys. Lett. 2000, 330, 169-174.

(32) O. Hino, Y. T.; Ten-no, S. Biorthogonal approach for explicitly correlated calculations using the transcorrelated Hamiltonian. J. Chem. Phys. 2001, 115, 7865-7871.

(33) Umezawa, N.; Tsuneyuki, S. Transcorrelated method for electronic systems coupled with variational Monte Carlo calculation. J. Chem. Phys. 2003, 119, 10015-10031.

(34) Umezawa, N.; Tsuneyuki, S.; Ohno, T.; Shiraishi, K.; Chikyow, T. A practical treatment for the three-body interactions in the transcorrelated variational Monte Carlo method: Application to atoms from lithium to neon. J. Chem. Phys. 2005, 122, 224101.

(35) Luo, H. Variational transcorrelated method. J. Chem. Phys. 2010, 133, 154109.

(36) Luo, H. Complete optimisation of multi-configuration Jastrow wave functions by variational transcorrelated method. J. Chem. Phys. 2011, 135, 024109.

(37) Yanai, T.; Chan, G. K.-L. Canonical transformation theory for multireference problems. J. Chem. Phys. 2006, 124, 194106.

(38) Yanai, T.; Shiozaki, T. Canonical transcorrelated theory with projected Slater-type geminals. J. Chem. Phys. 2012, 136, 084107.

(39) Grüneis, A.; Hirata, S.; Ohnishi, Y.; Ten-no, S. Perspective: Explicitly correlated electronic structure theory for complex systems. J. Chem. Phys. 2017, 146, 080901. 
(40) Booth, G. H.; Thom, A. J. W.; Alavi, A. Fermion Monte Carlo without fixed nodes: A game of life, death, and annihilation in Slater determinant space. J. Chem. Phys. 2009, 131, 054106.

(41) Cleland, D.; Booth, G.; Alavi, A. Communications: Survival of the fittest: Accelerating convergence in full configuration-interaction quantum Monte Carlo. J. Chem. Phys. 2010, 132, 041103.

(42) Booth, G. H.; Grüneis, A.; Kresse, G.; Alavi, A. Towards an exact description of electronic wavefunctions in real solids. Nature (London) 2013, 493, 365-370.

(43) Booth, G.; Cleland, D.; Thom, A.; Alavi, A. Breaking the carbon dimer: The challenges of multiple bond dissociation with full configuration interaction quantum Monte Carlo methods. J. Chem. Phys. 2011, 135, 084104.

(44) Kersten, J. A. F.; Booth, G. H.; Alavi, A. Assessment of multireference approaches to explicitly correlated full configuration interaction quantum Monte Carlo. J. Chem. Phys. 2016, 145, 054117.

(45) Booth, G.; Cleland, D.; Alavi, A.; Tew, D. An explicitly correlated approach to basis set incompleteness in full configuration interaction quantum Monte Carlo. J. Chem. Phys. 2012, 137, 164112.

(46) Torheyden, M.; Valeev, E. F. Universal perturbative explicitly correlated basis set incompleteness correction. J. Chem. Phys. 2009, 131, 171103.

(47) Kong, L.; Valeev, E. F. SF-[2](R12): A spin-adapted explicitly correlated method applicable to arbitrary electronic states. J. Chem. Phys. 2011, 135, 214105.

(48) Golub, G.; Loan, C. V. Matrix Computations, Third Edition; Johns Hopkins University Press, 1996. 
(49) Luo, H. Transcorrelated calculations of homogeneous electron gases. J. Chem. Phys. 2012, 136, 224111.

(50) Filippi, C.; Umrigar, C. J. Multiconfiguration wave functions for quantum Monte Carlo calculations of first-row diatomic molecules. J. Chem. Phys. 1996, 105, 213-226.

(51) Armour, E. A. G. The calculation of the ground-state energy of the free-electon gas by the transcorrelated method. J. Phys. 1980, C 13, 343-348.

(52) Gaskell, T. The Collective Treatment of a Fermi Gas: II. Proc. Phys. Soc. 1961, r7, $1182-1192$.

(53) Gaskell, T. The Collective Treatment of Many-body Systems: III. Proc. Phys. Soc. 1962, 80, 1091-1100.

(54) Kohn, W.; Sham, L. J. Self-Consistent Equations Including Exchange and Correlation Effects. Phys. Rev. 1965, A 140, 1133-1138.

(55) Ceperley, D.; Alder, B. J. Ground State of the electon Gas by a Stochastic Method. Phys. Rev. Lett. 1980, 45, 566-569.

(56) Fraser, L. M.; Foulkes, W. M. C.; Rajagopal, G.; Needs, R. J.; Kenny, S. D.; Williamson, A. J. Finite-size effects and Coulomb interactions in quantum Monte Carlo calculations for homogeneous systems with periodic boundary conditions. Phys. Rev. 1996, B53, 1814-1832.

(57) Kwon, Y.; Ceperley, D.; Martin, R. M. Effects of backflow correlation in the threedimensional electron gas: Quantum Monte Carlo study. Phys. Rev. 1998, B 58, 68006806.

(58) López Ríos, P.; Ma, A.; Drummond, N. D.; Towler, M. D.; Needs, R. J. Inhomogeneous backflow transformations in quantum Monte Carlo calculations. Phys. Rev. 2006, E $74,066701$. 
(59) Drummond, N. D.; Needs, R. J.; Sorouri, A.; Foulkes, W. M. C. Finite-size errors in continuum quantum Monte Carlo calculations. Phys. Rev. 2008, B 78, 125106.

(60) Shepherd, J. J.; Booth, G.; Grüneis, A.; Alavi, A. Full configuration interaction perspective on the homogeneous electron gas. Phys. Rev. 2012, B 85, 081103(R).

(61) Shepherd, J. J.; Booth, G. H.; Alavi, A. Investigation of the full configuration interaction quantum Monte Carlo method using homogeneous electron gas models. J. Chem. Phys. 2012, 136, 244101.

(62) Neufeld, V. J.; Thom, A. A study of the dense uniform electron gas with high orders of coupled cluster. J. Chem. Phys. 2017, 147, 194105.

(63) Petruzielo, F.; Holmes, A.; Changlani, H.; Nightingale, M.; Umrigar, C. Semistochastic Projector Monte Carlo Method. Phys. Rev. Lett. 2012, 109, 230201.

(64) Blunt, N.; Smart, S.; Kersten, J.; Spencer, J.; Booth, G.; Alavi, A. Semi-stochastic full configuration interaction quantum Monte Carlo: Developments and application. J. Chem. Phys. 2015, 142, 184107.

(65) Booth, G.; Smart, S.; Alavi, A. Linear-scaling and parallelisable algorithms for stochastic quantum chemistry. Molecular Physics 2014, 112, 1855-1869. 